\title{
Episodic and semantic autobiographical memory and everyday memory during late childhood and early adolescence
}

\author{
Karen A. Willoughby ${ }^{1,2}$, Mary Desrocher ${ }^{3}$, Brian Levine ${ }^{2,4}$ and Joanne F. Rovet ${ }^{1,2,5 *}$ \\ ${ }^{1}$ Neuroscience and Mental Health Program, The Hospital for Sick Children, Toronto, ON, Canada \\ 2 Department of Psychology, University of Toronto, Toronto, ON, Canada \\ ${ }^{3}$ Department of Psychology, York University, Toronto, ON, Canada \\ ${ }^{4}$ Rotman Research Institute, Baycrest Centre for Geriatric Care, Toronto, ON, Canada \\ ${ }^{5}$ Department of Pediatrics, University of Toronto, Toronto, ON, Canada
}

Edited by:

Susan M. Rivera, University of California, USA

Reviewed by:

Olivier Pascalis, CNRS and University

Pierre Mendes France, France

Bradley Love, University College

London, UK

*Correspondence:

Joanne F. Rovet, Neuroscience and

Mental Health Program, The Hospital

for Sick Children, 555 University

Avenue, Toronto, ON, Canada M5G

$1 \times 8$.

e-mail: joanne.rovet@sickkids.ca
Few studies have examined both episodic and semantic autobiographical memory (AM) performance during late childhood and early adolescence. Using the newly developed Children's Autobiographical Interview (CAl), the present study examined the effects of age and sex on episodic and semantic AM and everyday memory in 182 children and adolescents. Results indicated that episodic and semantic AM both improved between 8 and 16 years of age; however, age-related changes were larger for episodic AM than for semantic AM. In addition, females were found to recall more episodic AM details, but not more semantic AM details, than males. Importantly, this sex difference in episodic AM recall was attenuated under conditions of high retrieval support (i.e., the use of probing questions). The ability to clearly visualize past events at the time of recollection was related to children's episodic AM recall performance, particularly the retrieval of perceptual details. Finally, similar age and sex effects were found between episodic AM and everyday memory ability (e.g., memory for everyday activities). More specifically, older participants and females exhibited better episodic AM and everyday memory performance than younger participants and males. Overall, the present study provides important new insight into both episodic and semantic AM performance, as well as the relation between episodic AM and everyday memory, during late childhood and adolescence.

Keywords: autobiographical memory, everyday memory, sex differences, childhood, adolescence, development

\section{INTRODUCTION}

Autobiographical memory (AM), or the recollection of specific personal events, is a multifaceted higher-order cognitive process that includes both episodic and semantic memory components (Levine et al., 2002; Tulving, 2002). Episodic AM refers to remembering past events that are specific in time and place and it typically involves the recollection of vivid sensory, perceptual, and emotional details (e.g., my last birthday; Tulving, 2002; Addis et al., 2004). One additional critical component of episodic AM that differentiates it from other forms of declarative memory is the requirement for autonoetic consciousness or the ability to travel back in time mentally in order to re-experience the event at time of recollection (Wheeler et al., 1997; Tulving, 2002). Semantic AM, in contrast, refers to the recollection of personal facts, traits, or general self-knowledge, which are independent of time, place, and any sense of re-experiencing a past event (e.g., I am 30 years old; Levine et al., 2002; Tulving, 2002). Episodic AM and semantic AM are highly interconnected, especially during the early stages of retrieval when personal semantic knowledge can aid memory search and retrieval operations (Conway and Pleydell-Pearce, 2000; Svoboda et al., 2006). However, recent research suggests that these two forms of AM can be differentiated, not only by their distinct properties, but also by their developmental trajectories (Piolino et al., 2007) and their underlying neural activations (Maguire and Mummery, 1999; Levine et al., 2004). More specifically, episodic AM retrieval has been associated with greater activity in the hippocampus (Maguire and Mummery, 1999; Hoscheidt et al., 2010), as well as a later and more gradual developmental trajectory (Tulving, 2002; Piolino et al., 2007) than semantic AM retrieval.

Due to infants' and young children's undeveloped language skills, it is often difficult to investigate the emergence and characteristics of episodic and semantic AM in the first few years of life. Typically, early investigations into the emergence of episodic AM relied on retrospective studies requiring adults to recall and date their earliest AMs. The results of these studies indicated that few AMs predated the age of 2 years with the majority of adults' earliest memories clustering around the ages of 3 or 4 (Bruce et al., 2000; Rubin, 2000). More recently, Newcombe et al. (2007) have proposed that the inability to recall early childhood events can be classified into two distinct periods of amnesia: (i) infantile amnesia, which encompasses approximately the first 2 years of life, refers to the complete absence of episodic AM, and (ii) childhood amnesia, which refers to a period of impoverished episodic AM 
that gradually improves between 3 and 5 years of age (Perner and Ruffman, 1995; Newcombe et al., 2007).

During the period of infantile amnesia, evidence suggests that infants develop early forms of semantic memory before they develop episodic AM. For instance, studies using deferred imitation (e.g., repeating a series of actions following a delay) have shown that infants as young as 6 months of age exhibit early forms of declarative memory, as they are able to retain contextual information about object-action events after a delay (Meltzoff, 1995; Barr et al., 1996). Because infants often generalize this newly learned information to other domains and do not appear to spontaneously re-experience the past learning episode (i.e., reflecting autonoetic consciousness) or integrate it into the temporal continuity of their lives, this early form of declarative memory may be classified as being more semantic in nature rather than episodic (Bauer and Dow, 1994; Lechuga et al., 2001; Nelson and Fivush, 2004; Newcombe et al., 2007). In addition, Fivush and Hamond (1990) showed that 2- to 4-year-old children can retain specific knowledge of past events (e.g., a school trip) even after a lengthy delay period. However, this early event knowledge may represent only a rudimentary form of AM because it is often fragmentary, semantic, and heavily dependent on the provision of retrieval cues or prompting questions by adults (Fivush and Hamond, 1990; Perner and Ruffman, 1995; Newcombe et al., 2007). Overall, very young children (i.e., $<4$ years of age) are clearly able to use their personal experiences in order to acquire new event-related information and develop their semantic autobiographical knowledge base. However, they are not yet fully capable of spontaneously recalling and re-experiencing past autobiographical events.

The apparent absence of episodic AM during the period of infantile amnesia is thought to be due to immature hippocampal functioning, particularly poor encoding and storage of details associated with specific events (Bauer, 2006; Newcombe et al., 2007). The hippocampus is a medial temporal lobe structure that is known to play a crucial role in the formation, consolidation, and retrieval of episodic AMs (Eldridge et al., 2000; Ryan et al., 2001; Moscovitch, 2008). More specifically, the hippocampus binds patterns of neural activity present at the time of encoding into a memory trace that can be sustained and retained over time (Eichenbaum and Bunsey, 1995; Eichenbaum, 2000; Buckner and Wheeler, 2001). To date, numerous lesion studies have shown that patients with hippocampal damage exhibit deficits in episodic AM, but not semantic AM, indicating that episodic AM is more critically dependent on the hippocampus than semantic AM (Viskontas et al., 2000; Maguire et al., 2001; Addis et al., 2007; St-Laurent et al., 2009). According to Bachevalier and VarghaKhadem (2005), most abilities that depend on the hippocampus, such as episodic AM, emerge late in development because the hippocampus continues to grow during the first 2 years of life and has substantial postnatal maturation. Thus, several investigators have proposed a hierarchical model of AM with semantic AM developing early and providing a foundation for the later and more gradual development of episodic AM (Mishkin et al., 1997).

The shift from infantile to childhood amnesia, as well as a later shift to more mature levels of AM functioning, appear to be associated with several factors. These include improvements in the general functioning of basic memory processes (i.e., encoding, storage, and retrieval), maturation of requisite brain regions and neural networks, and advances in higher-order cognitive processes that facilitate episodic AM and autonoetic consciousness (e.g., language, self-awareness, theory of mind, executive functions, etc., Hudson and Fivush, 1991; Perner and Ruffman, 1995; Howe et al., 2003; Picard et al., 2009). For instance, Howe and Courage (1993, 1997) have demonstrated, using self-recognition mirror tests, that the emergence of episodic AM and the offset of infantile amnesia correspond with the development of the cognitive self. This knowledge structure enables children to recognize that the self is continuous over time, having a past and a future, which then allows them to organize and integrate personally experienced events within their self-concept (Howe et al., 2003). Without this form of self-awareness, children are unable to encode and store events as subjective experiences that are integrated within the personal self (Wheeler et al., 1997). Thus, a fully developed cognitive self seems to be an important prerequisite for the initial emergence of episodic AM, as children cannot encode, store, or retrieve events as personally relevant without this capacity.

Similarly, Perner and Ruffman (1995) have argued that it is not until children experience advances in metacognition (i.e., theory of mind) around the age of 4 that they begin to demonstrate true episodic AM, as opposed to simply knowing something about a past event. According to this perspective, children are unable to fully re-experience past events at the time of recollection (i.e., they cannot demonstrate autonoetic consciousness) until they first acquire the ability to understand their own mental states in the past and future (Perner and Ruffman, 1995). Finally, the gradual improvement of episodic AM between 3 and 5 years of age may also be associated with maturation of the requisite brain regions and neural networks involved in AM, particularly the hippocampus and prefrontal cortex (Wheeler et al., 1997; Gogtay et al., 2006; Newcombe et al., 2007). Similar to the hippocampus, the prefrontal cortex has been identified as a critical region for AM retrieval because it controls self-referential processing (i.e., processing personal information), as well as memory search, retrieval, and evaluation processes, through its interactions with the hippocampus and medial temporal lobe (Buckner and Wheeler, 2001; Svoboda et al., 2002; Cabeza et al., 2004). Given that the hippocampus and prefrontal cortex both exhibit prolonged structural and functional maturation during childhood and adolescence (Benes et al., 1994; Giedd et al., 1996; Huttenlocher and Dabholkar, 1997; Suzuki et al., 2005; Gogtay et al., 2006; Lenroot and Giedd, 2006; Grieve et al., 2011), it is possible that episodic AM continues to develop during late childhood and adolescence due to improved neural connectivity between the hippocampus, prefrontal cortex, and other core regions of the AM neural network.

Despite an extensive literature on the emergence and development of episodic AM across early childhood (e.g., Pillemer et al., 1994; Van Abbema and Bauer, 2005; Bauer et al., 2007), only two studies to date have examined age-related changes in both episodic and semantic AM beyond the age of 5. For example, Piolino et al. (2007) examined episodic and semantic AM in 42 children between the ages of 7 and 13 using the TEMPAu task, which is an adapted version of the Autobiographical Memory Interview (AMI; Kopelman et al., 1989). The TEMPAu task assesses children's recollection of specific personal events (e.g., a 
school event, trip, or vacation, and a family event) from three different time periods (e.g., current school year, last school year, and previous school years; Piolino et al., 2007). Using this measure, Piolino et al. (2007) found significant age-related improvements in episodic AM, but not semantic AM (e.g., recalling names of childhood friends), across childhood. In a second more recent study by the same research team, the TEMPAu task was used to examine AM performance in 30 children between 6 and 11 years of age (Picard et al., 2009). Contrary to Piolino et al.'s (2007) results, however, Picard et al. (2009) now observed that both episodic and semantic AM performance increased with age. This discrepancy between studies may be due to the use of different statistical analyses for examining age effects across the two studies. For example, Piolino et al. (2007) stratified their sample into three age groups (e.g., 78, 9-10, and 11-13) that were compared using ANOVA, whereas Picard et al. (2009) examined age as a continuous variable in a regression analysis, which may be a more sensitive measure of agerelated changes in semantic AM. Thus, it appears that age-related changes in both episodic and semantic AM components are evident during childhood. Additionally, Picard et al. (2009) found that age-related improvements in episodic AM across childhood were associated with age-related improvements in semantic AM, general episodic memory, and executive functioning (Picard et al., 2009). These findings suggest that the development of episodic AM corresponds to the development of other cognitive abilities, including those that rely on the hippocampus (i.e., general episodic memory) and prefrontal cortex (i.e., executive functioning; Picard et al., 2009).

Both Piolino et al.'s (2007) and Picard et al.'s (2009) studies provided novel data on episodic and semantic AM during childhood, however, their assessment of episodic and semantic AM was based on separate tasks that were not matched for psychometric properties. For example, episodic AM was measured using the TEMPAu task, in which examiners provided subjective ratings (i.e., on a four-point scale) of the overall specificity of participants' recollections of childhood events (Piolino et al., 2007). In contrast, semantic AM was measured using a questionnaire requiring participants to list personal facts (e.g., childhood heroes, home address, names of friends, and hobbies; Piolino et al., 2007). Thus, it may be difficult to make direct comparisons of age-related changes in episodic and semantic AM from these two studies due to possible task-related confounds (Levine, 2004). Given that episodic AMs are often embedded within a semantic context that links episodic AM details with personal facts, extended events, and lifetime periods (Svoboda et al., 2006), it would be particularly informative to examine whether the number of episodic and semantic AM details recalled within a single autobiographical narrative change across childhood and adolescence. Thus, further replication of these studies using an objective measure of AM that effectively dissociates episodic AM from semantic AM within a single task is warranted. In addition, no study has yet investigated age-related changes in episodic and semantic AM performance across both childhood and adolescence (i.e., beyond the age of 13).

Thus, the main goal of the present study was to collect normative data on age-related changes in both episodic and semantic AM across childhood and adolescence using the Children's Autobiographical Interview (CAI), an adaptation of Levine et al.'s (2002)
Autobiographical Interview. In contrast to the TEMPAu task, the CAI: (a) effectively dissociates episodic and semantic AM within a single autobiographical narrative, (b) provides an objective and reliable measure of episodic AM (i.e., total number of details recalled), which can be subdivided into specific subcategories of details, (c) incorporates a standardized qualitative rating system as an additional measure of episodic re-experiencing that is comparable to the rating system used in the TEMPAu task, and (d) allows for the examination of different levels of retrieval support (Levine et al., 2002). Although the Autobiographical Interview has been used extensively to examine episodic and semantic AM in healthy young adults, older adults, and patients with hippocampal damage (Levine et al., 2002; Steinvorth et al., 2005; Addis et al., 2007; St-Laurent et al., 2009), it has not yet been used to examine AM performance in children or adolescents. Thus the present study sought to provide normative child data from the CAI so that this measure can be used by future studies to explore AM in typically and atypically developing pediatric populations. Based on Piolino et al.'s (2007) and Picard et al.'s (2009) results in children, it was hypothesized that both episodic and semantic AM performance would improve across childhood and adolescence; however, greater age-related improvements would be seen in episodic AM than in semantic AM during this developmental period.

A second goal of the present study was to compare episodic and semantic AM performance in males versus females across childhood and adolescence. Previous research examining sex differences in $\mathrm{AM}$ in adults and young children has shown that females tend to have better episodic AM than males, as they provide more detailed, accurate, vivid, and emotional recollections (Ross and Holmberg, 1992; Buckner and Fivush, 1998; Davis, 1999; Pillemer et al., 2003). These sex differences appear to be specific to the episodic component of AM because males and females perform similarly on tests of general semantic memory (Bahrick et al., 1975; Herlitz et al., 1997). However, no study has yet specifically examined sex differences in semantic AM. Interestingly, Bloise and Johnson (2007) suggested that one reason why females tend to outperform males in episodic memory recall tasks is because they may have more efficient memory retrieval and organizational processes than males. For example, women outperformed men when asked to recall episodic information from a narrative, but not when their recognition memory for the same information was tested (Bloise and Johnson, 2007). Given that Bloise and Johnson's (2007) study showed that sex differences in episodic memory can be eliminated in the context of high retrieval support (i.e., in the recognition task), it is possible that sex differences in episodic AM across childhood and adolescence may similarly be reduced under conditions of high retrieval support, in which the use of retrieval strategies is less critical. Based on previous findings, we expected that females would outperform males in episodic AM, but not semantic, AM, particularly under conditions of low retrieval support.

Finally, given that Picard et al. (2009) showed parallel improvements between episodic AM and several other cognitive functions across childhood (e.g., general episodic memory), we were also interested in examining whether similar age and sex effects were evident between episodic AM and everyday memory, both of which rely on the hippocampus (Vargha-Khadem et al., 1997; Maguire and Mummery, 1999). Everyday memory, as measured 
by the everyday memory questionnaire (EMQ; Sunderland et al., 1983), reflects one's ability to perform real-world memory tasks within various domains, such as remembering faces, places, and actions, as well as learning new tasks. Several studies have shown that children with early hippocampal damage exhibit deficits in both general episodic memory and everyday memory using the EMQ (Vargha-Khadem et al., 1997; Isaacs et al., 2000; Willoughby et al., 2008). However, research on everyday memory and its relation to episodic AM in typically developing children is limited. One study by Drysdale et al. (2004) examined everyday memory performance in 226 typically developing 5- to 12-year-old children using a modified version of the EMQ, but found no significant age or sex differences. The absence of age-related differences within this sample may reflect the fact that their EMQ data were positively skewed across the age distribution, leading the authors to conclude that the EMQ is better suited as a test of everyday memory ability for older children and adolescents than for young children (Drysdale et al., 2004). Clearly, further investigation of the effects of age and sex on everyday memory during late childhood and adolescence is required. Based on Picard et al.'s (2009) findings, we expected to find similar age and sex effects between episodic AM and everyday memory in typically developing children and adolescents.

\section{MATERIALS AND METHODS PARTICIPANTS}

Two hundred twenty-three typically developing children and adolescents (52\% male) between 8 and 16 years of age $(M=11.80$, $\mathrm{SD}=2.55)$ were recruited for the present study while visiting the Ontario Science Centre in Toronto, ON, Canada. Exclusionary criteria included a diagnosis of attention-deficit hyperactivity disorder (ADHD), an identified learning disability, a psychiatric disorder, a head injury resulting in a loss of consciousness, or a debilitating or chronic medical condition. As a result, 17 participants were excluded for having ADHD and 8 for having a learning disability. In addition, 16 participants were unable to recall two specific AMs (e.g., they described a week long event) and their data were excluded from the analyses (note: these cases were equally distributed across age groups). Thus, the final sample of participants consisted of 182 children and adolescents (48\% male) between 8 and 16 years of age $(M=11.88, \mathrm{SD}=2.55)$. The distribution of participants by age and sex is presented in Table 1 . English was the primary language spoken in all homes; however, $14 \%$ of participants were also fluent in a second language (e.g., Chinese, Arabic, Greek, or Tamil). Socioeconomic status (SES) of each participant was computed using the Hollingshead FourFactor Index (Hollingshead, 1975) based on parental occupation and education. Family Hollingshead SES scores ranged from 25 to $66(M=48.70, \mathrm{SD}=10.67)$ and included medium-low $(3 \%)$, medium (22\%), medium-high (40\%), and high (35\%) income groups.

\section{GENERAL PROCEDURE}

All parents or guardians provided written informed consent for their child's participation in this study while participants also provided verbal informed assent. Interviews were conducted on eight successive weekends over a 2 -month period. Participants were interviewed individually for approximately $20 \mathrm{~min}$ in an enclosed quiet area of the Ontario Science Centre, while parents/guardians also completed two questionnaires. One extensively trained examiner (Karen A. Willoughby) conducted $53 \%$ of the interviews, while four other trained examiners conducted $24,13,7$, and $3 \%$ of the interviews respectively. An exploration of the data revealed that there were no significant differences between the examiners in terms of the number of details recalled, $p>0.05$. Upon completion of the interview, participants received a certificate of participation and a pencil. All procedures were approved by the Human Participants Review (Ethics) Sub-Committee of York University and the Research Ethics Board of the Hospital for Sick Children.

\section{DEMOGRAPHIC INFORMATION}

Parents/guardians completed a detailed demographic questionnaire that yielded information about the child's age, sex, ethnicity, primary language, overall health, psychiatric health, the parents' marital status, and family SES. Nine parents/guardians chose not to complete the demographic questionnaire but did indicate their child's age and sex.

\section{CHILDREN'S AUTOBIOGRAPHICAL INTERVIEW}

The CAI was developed for the present study and is an adapted child-friendly version of Levine et al.'s (2002) Autobiographical Interview for adults. For this task, participants were required to recall two AMs that occurred at a specific time and place more than 1 month previously (see Appendix for instructions). In order to assist with memory retrieval, participants were shown a list of 18 sample autobiographical events (e.g., your last birthday), but were also told that they did not have to choose an event from that list.

The CAI has three distinct phases: free recall, general probe, and specific probe. In the free recall phase, participants described as many details as they could remember about the event without interruption until either it became clear that the end of their description was reached or $5 \mathrm{~min}$ had elapsed. After the initial description of the event, general probes were provided in order to encourage recall of additional details (e.g., "Is there anything

Table 1 | Number of males and females at each age group.

\begin{tabular}{lcccccrrrr}
\hline & \multicolumn{9}{c}{ Age } \\
\cline { 2 - 8 } & $\mathbf{8}$ & $\mathbf{9}$ & $\mathbf{1 0}$ & $\mathbf{1 1}$ & $\mathbf{1 2}$ & $\mathbf{1 3}$ & $\mathbf{1 4}$ & $\mathbf{1 5}$ & $\mathbf{1 6}$ \\
\hline Number of males & 10 & 11 & 11 & 11 & 12 & 10 & 8 & 11 \\
Number of females & 10 & 11 & 8 & 12 & 12 & 10 & 8 & 8 \\
Total number of participants & 20 & 22 & 19 & 23 & 24 & 20 & 16 & 19
\end{tabular}


else you can tell me about that event?") or to narrow down overly general or multiple event descriptions into a single event (e.g., "You described a couple of events. I would like you to only tell me about one of those events. Choose the one that you feel you remember the best"). If participants were unable to produce a specific event after general probing, they were given the opportunity to choose a different event to describe. Finally, in the specific probe phase, participants answered a series of standardized questions that served to promote retrieval of any episodic details that were not recalled in the previous phases (e.g., "When did this event take place?"). In order to prevent the specific probe process from contaminating free recall of the second event, specific probing was administered after both events had been described under both free recall and general probe phases.

Finally, after the specific probing phase, participants provided self-report ratings for both AMs on the following seven aspects: (i) whether they experienced a change in emotions from before to after the event $(1=$ no change, $7=$ lots of change $)$; (ii) personal importance at time of testing $(1=$ not important, $7=$ most important event); (iii) personal importance at time of event $(1=$ not important, 7 = most important event); (iv) amount of rehearsal $(1=$ once every few years, $7=$ every day $) ;(\mathrm{v})$ visualization of event $(1=$ ca not see it at all, $7=$ really clear $)$; (vi) strength of memory ( $1=$ not strong, $7=$ really strong); and (vii) confidence about the recollection $(1=$ not sure, $7=$ really sure $)$.

\section{SCORING PROTOCOL FOR THE CAI}

Each participant's tape-recorded interview was transcribed and scored according to the Autobiographical Interview Scoring Manual (Levine et al., 2002). The transcribed text was segmented into two main categories of details: internal (episodic) and external (non-episodic). Details were defined as episodic if they were directly related to the event described, were specific in time and place, and conveyed a sense of episodic re-experiencing. These details were then assigned to one of five episodic detail subcategories: (a) event, (b) place, (c) time, (d) perceptual, and (e) emotion/thought (see Figure 1 for a scoring example). The remaining details were considered non-episodic and were assigned to one of four non-episodic detail subcategories: (a) semantic facts, (b) unsolicited repetitions of previously recalled details, (c) other metacognitive statements, and (d) external event details unrelated to the main event recalled (see Figure 1 for examples). Details were then tallied for each episodic and non-episodic subcategory and summed to form total episodic and total non-episodic detail composite scores across each phase of the CAI (e.g., free recall,

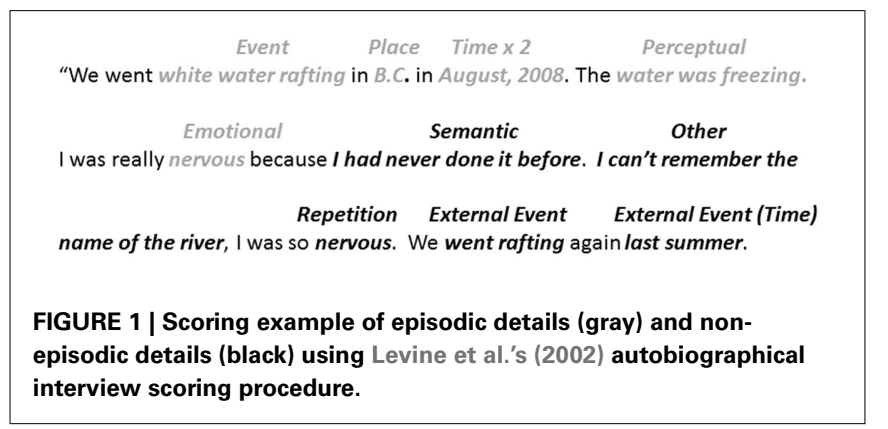

general probe, and specific probe). Each AM was also assigned a total experimenter rating composite, which assessed the overall quality of episodic re-experiencing (i.e., on a scale from 0 to 18), and a time integration rating, which assessed participants' ability to integrate the event into a larger time scale or life history (i.e., on a scale from 0 to 3; see Levine et al., 2002, for a more detailed description of the experimenter ratings). For each participant, the AM detail scores, experimenter ratings, and participant self-report ratings were averaged across their two AMs.

All memories were first scored by Karen A. Willoughby and then for reliability, re-scored by a second trained individual, and both scorers were blind to participants' age and sex, and had undergone extensive training using a practice set of 20 AMs. Inter-rater reliability was assessed using intra-class correlations (one-way random effects model; McGraw and Wong, 1996). Coefficients for total episodic and total non-episodic details were 0.92 and 0.98 for free recall, 0.97 and 0.90 for general probe, and 0.96 and 0.89 for specific probe. Coefficients for the episodic subcategories ranged from 0.74 to 0.95 , and coefficients for the non-episodic subcategories ranged from 0.78 to 0.83 . Agreement on the experimenter rating composite was 0.80 for free recall, 0.79 for general probe, but it was lower for specific probe (0.62) due to the limited range since most participants received maximum scores after specific probing.

\section{EVERYDAY MEMORY QUESTIONNAIRE}

Parents/guardians completed the (EMQ; Sunderland et al., 1983), which was comprised of 28 items assessing any difficulties in their child's memory ability in everyday life situations along a ninepoint scale ranging from 1 (not at all in the last 3 months) to 9 (more than once a day). Lower EMQ scores signified better everyday memory. Using a factor structure developed by Cornish (2000), three composite scores were created: (a) memory for everyday activities (i.e., from five items, e.g., "forgets a change in his/her daily routine"), (b) spatial everyday memory (i.e., from four items, e.g., "gets lost where he/she has often been before"), and (c) a total EMQ composite based on the square root of the sum of all 28 items. Twenty-six parents/guardians did not complete the EMQ; however, these missing cases were equally distributed across age groups.

\section{DATA ANALYSIS}

Given that very few additional details from the CAI were recalled during the general probe phase, details from the first two phases (i.e., free recall and general probe) were collapsed to form a single "Recall" condition. This Recall condition was analyzed separately from a "Recall + Specific Probe" condition, which comprised participants' cumulative scores across all three phases of the CAI. Similar to Levine et al. (2002), the specific probe phase was not examined in isolation because it would have penalized participants who provided richly detailed accounts in the free recall phase and consequently, received fewer probing questions in the specific probe phase. In addition, direct statistical comparisons between scores in the Recall condition and the Recall + Specific Probe condition could not be conducted because these scores were highly correlated. Therefore, in order to examine the effects of retrieval support, we assessed whether any significant effects found in the 
Recall condition were reduced to non-significant effects in the Recall + Specific Probing condition (i.e., following the addition of probing questions).

First, all variables of interest were examined for outliers (i.e., $>3$ SD from the mean) and any outliers found were replaced with the highest or lowest reasonable score using Winsorization procedures (note: results were identical when outliers were not replaced). In addition, all variables were examined for deviations in skewness and kurtosis in order to assess the assumption of normality and any variable that exceeded the cutoffs $( \pm 2)$ was corrected using $\log 10$ transformation. In all analyses, exact chronological age (in days) was used. Although the overall significance level was set at $p<0.01$ in order to control for a large number of regression analyses conducted, a few important trend-level sex differences (at $p<0.05$ ) were found and these will be discussed below. Given that the demographic variables (e.g., SES, ethnicity) and examiner had no effect on the results when used as covariates in each of the regression analyses, these variables were not considered further.

In order to examine the effects of age and sex on episodic and semantic AM performance, hierarchical linear regressions were conducted using retention interval (i.e., delay since the event) as a control variable in step one, sex as the first predictor in step two, age as the second predictor in step three, and the ageby-sex interaction variable in step four. Retention interval (i.e., $M=1.97$ years; $\mathrm{SD}=1.95$ years; averaged across the two AMs) was used as a control variable because it was negatively correlated with the number of event details, $r=-0.158, p=0.033$, and time details, $r=-0.166, p=0.025$, recalled in the Recall + Specific Probe condition. Pearson correlations were used to determine whether participants' self-report ratings of their AM recollections were associated with the number of episodic AM details recalled in the Recall + Specific Probe condition. Finally, to examine the effects of age and sex on everyday memory, hierarchical linear regressions were used, with sex as the first predictor in step one, age as the second predictor in step two, and the age-by-sex interaction variable in step three. All variables were standardized before being entered in each regression analysis.

\section{RESULTS \\ EFFECTS OF AGE AND SEX ON AM PERFORMANCE IN THE RECALL CONDITION}

First, we examined whether age and sex predicted total episodic and total non-episodic details in the Recall condition. For the complete set of regression results please refer to Tables A1 and A2 in Appendix. Age significantly predicted both total episodic, $\Delta F=36.71 ; \Delta R^{2}=0.17 ; B=0.42 ; p<0.001$, and total nonepisodic details, $\Delta F=13.48 ; \Delta R^{2}=0.07 ; B=0.26 ; p<0.001$; however age accounted for a greater proportion of the variance in total episodic details, $\Delta R^{2}=0.17$, than in total non-episodic details, $\Delta R^{2}=0.07$ (note: a $t$-test revealed that the correlation coefficient between age and total episodic details, $r=0.402$, was significantly greater than the correlation coefficient between age and total non-episodic details, $r=0.268$; $t=2.12, p<0.05)$. Generally, older participants recalled more episodic and non-episodic details than did younger participants (see Figure 2A for means and SEs across age). A trend-level effect of sex was found only for total episodic details, $\Delta F=5.13 ; \Delta R^{2}=0.03 ; B=0.17 ; p<0.05$,

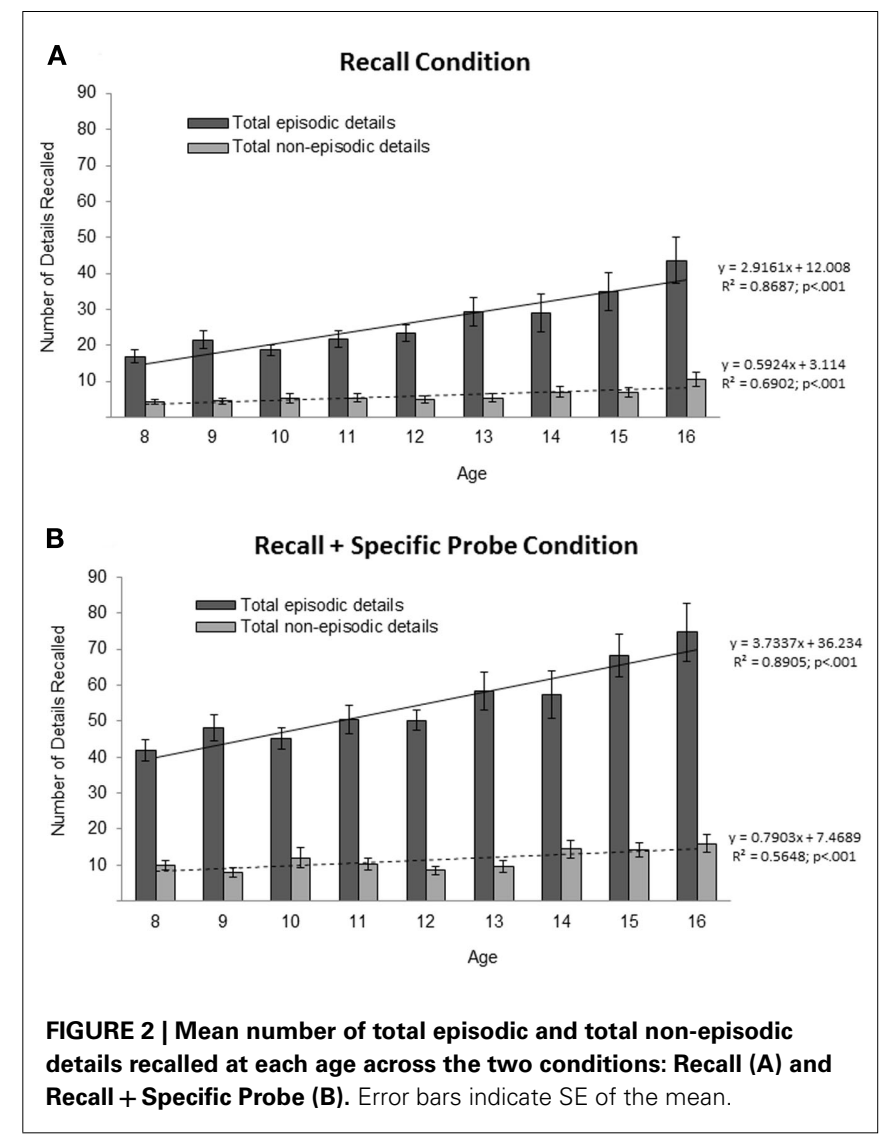

with females reporting more details than males (see Figure 3A for means and SEs across sex).

Next, we examined the effects of age and sex on each episodic detail subcategory (i.e., event, place, time, perceptual, and emotion/thought), as well as each non-episodic detail subcategory (i.e., semantic details, repetitions, other metacognitive statements, and external event details). Age significantly predicted all five episodic details subcategories [ $p s<0.001$; see Table A1 (part 2) in Appendix for regression results] and three of the four nonepisodic detail subcategories (i.e., semantic details at $p<0.01$, repetitions at $p<0.001$, and other metacognitive statements at $p<0.001$; see Table A1 (part 3) in Appendix for regression results), with older participants recalling more details than younger participants. Importantly, age accounted for a greater proportion of the variance for all episodic subcategories than for all nonepisodic subcategories, including semantic details (see Table A1 in Appendix for $\Delta R^{2}$ values). Sex was found to predict the number of event, time, and perceptual details recalled in the Recall condition at trend levels ( $p s<0.05$; see Table A1 (part 2) in Appendix for regression results). In addition, trend-level age-bysex interactions were found for perceptual details, $\Delta F=4.17$; $\Delta R^{2}=0.02 ; B=0.14 ; p<0.05$, and other metacognitive statements, $\Delta F=6.12 ; \Delta R^{2}=0.03 ; B=0.14 ; p<0.05$. Post hoc comparisons indicated that for females only, age significantly predicted perceptual details, $F(1,91)=18.84, p<0.001$, and other metacognitive statements, $F(1,91)=16.32, p<0.001$, with older females ( $\geq$ age 13 ) reporting more details than younger females (<age 13). 

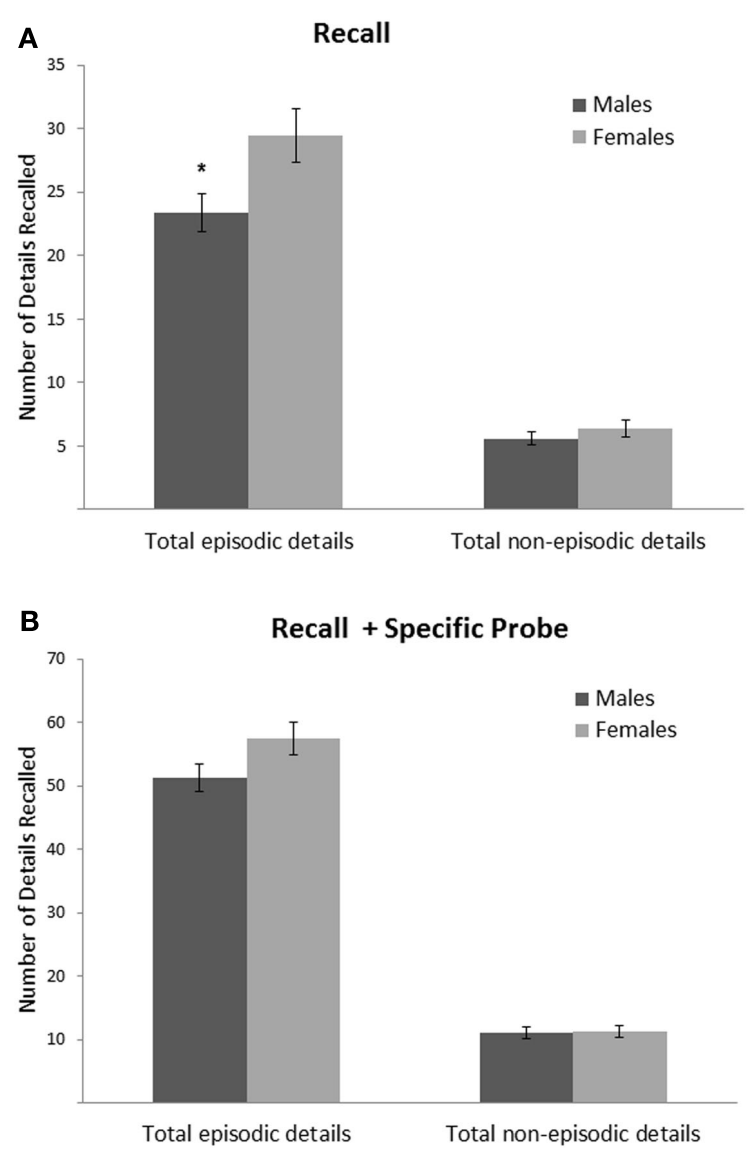

FIGURE 3 | Mean number of total episodic and total non-episodic details recalled for each sex across the two conditions: Recall $(A)$ and Recall + Specific Probe (B). Error bars indicate SE of the mean; ${ }^{*} p<0.05$.

\section{EFFECTS OF AGE AND SEX ON AM PERFORMANCE IN THE RECALL + SPECIFIC PROBE CONDITION}

A similar series of analyses were conducted to examine the effects of age and sex on AM performance in the Recall + Specific Probe condition, after accounting for the provision of high retrieval support (see Table A2 in Appendix for the full set of regression results. Age significantly predicted the total episodic details, $\Delta F=36.46$; $\Delta R^{2}=0.17 ; B=0.40 ; p<0.001$, and total non-episodic detail composites, $\Delta F=11.34 ; \Delta R^{2}=0.06 ; B=0.24 ; p<0.001$ (see Figure $2 \mathbf{B}$ for means and SEs across age). In addition age significantly predicted all five episodic subcategories $(p s<0.001)$, and three out of the four non-episodic details (i.e., semantic details at $p<0.01$, repetitions at $p<0.01$, and other metacognitive statements at $p<0.001$; see Table A2 in Appendix for regression results). Again, older participants recalled more details than younger participants and age accounted for a greater proportion of the variance for the episodic detail composite and subcategories scores than for the non-episodic detail composite and subcategories scores (i.e., including semantic details; see Table A2 in Appendix for $\Delta R^{2}$ values). In addition, a $t$-test revealed that the correlation coefficient between age and total episodic details in the Recall + Specific Probe condition, $r=0.368$, was significantly greater than the correlation coefficient between age and total non-episodic details, $r=0.238 ; t=2.07, p<0.05$.

Unlike the results from the Recall condition, sex no longer predicted total episodic, event, time, or perceptual details in the Recall + Specific Probe condition, indicating that sex differences in episodic AM were reduced after the provision of high retrieval support (see Figure 3B). However, sex was found to predict the number of emotion/thought details recalled in the Recall + Specific Probe condition, $\Delta F=5.45 ; \Delta R^{2}=0.03 ; B=0.15 ; p<0.05$, with females reporting more emotion/thought details than males. Furthermore, similar to the Recall condition, a trend-level age-bysex interaction was found for other metacognitive statements, $\Delta F=6.12 ; \Delta R^{2}=0.03 ; B=0.14 ; p<0.05$. Post hoc comparisons indicated that age significantly predicted other metacognitive statements, $F(1,91)=11.05, p=0.001$, in females only, with older females ( $\geq$ age 13$)$ reporting more details than younger females (<age 13).

The effects of age and sex on the experimenter rating composite and the time integration rating were also examined [see Table A2 (part 4) in Appendix]. Age significantly predicted the experimenter rating composite, $\Delta F=22.20 ; \Delta R^{2}=0.11$; $B=0.34 ; p<0.001$, and the time integration rating, $\Delta F=35.09$; $\Delta R^{2}=0.016 ; B=0.42 ; p<0.001$. Older participants received higher ratings of the overall quality of their AM recollections and their ability to integrate each AM into a larger time scale or life history than younger participants. No significant effect of sex was found.

\section{Correlations between participants' self-report ratings and episodic}

\section{AM performance}

Pearson correlations were used to examine whether participants' self-report ratings of their AM recollections were associated with their episodic AM performance (i.e., the number of episodic details recalled in the Recall + Specific Probe condition). As shown in Table 2, participants who rated themselves higher in the ability to visualize each event at the time of recollection recalled more total episodic, time, and perceptual details in the Recall + Specific Probe condition. Participants' ratings of the strength of their AMs were also positively associated with the number of total episodic, event, time, and perceptual details recalled. Finally, the more confident participants were about the accuracy of their recollections, the greater the number of perceptual details and emotion/thought details recalled.

\section{EVERYDAY MEMORY AND EPISODIC AM}

Finally, to determine whether similar effects would be found in everyday memory as those observed in episodic AM recall, the effects of age and sex on everyday memory performance were examined using hierarchical linear regressions. Results indicated that age significantly predicted both the EMQ composite, $\Delta F(1,153)=7.33, B=-0.21, p=0.008$, and the subscales representing spatial everyday memory, $\Delta F(1,153)=10.25, B=-0.24$, $p=0.002$, and memory for everyday activities, $\Delta F(1,153)=6.99$, $B=-0.21, p=0.009$. In each case, older participants had lower scores (i.e., better everyday memory ability) than younger participants (see Table 3A for means and SD across age). In addition, sex significantly predicted the EMQ composite, $\Delta F(1,154)=6.16$, 
Table 2 | Correlations between participants' self-report ratings and episodic AM performance in the recall + specific probe condition.

\begin{tabular}{|c|c|c|c|c|c|c|c|}
\hline Variable & $\begin{array}{l}\text { Emotion } \\
\text { change }\end{array}$ & $\begin{array}{l}\text { Importance } \\
\text { now }\end{array}$ & $\begin{array}{l}\text { Importance } \\
\text { then }\end{array}$ & $\begin{array}{l}\text { Amount of } \\
\text { rehearsal }\end{array}$ & $\begin{array}{l}\text { Visualization of } \\
\text { event }\end{array}$ & $\begin{array}{l}\text { Strength of } \\
\text { memory }\end{array}$ & $\begin{array}{l}\text { Confidence of } \\
\text { memory }\end{array}$ \\
\hline Total episodic details & 0.13 & 0.02 & 0.03 & 0.12 & $0.20 * *$ & $0.22 * *$ & $0.17^{*}$ \\
\hline Place & 0.10 & -0.12 & -0.03 & -0.00 & 0.14 & $0.16^{*}$ & 0.05 \\
\hline Time & 0.15 & 0.04 & 0.12 & 0.12 & $0.22 * *$ & $0.19 * *$ & $0.16^{*}$ \\
\hline Perceptual & 0.12 & 0.05 & -0.02 & 0.10 & $0.24 * * *$ & $0.27 * * *$ & $0.22 * *$ \\
\hline
\end{tabular}

${ }^{*} p<0.05,{ }^{*} p<0.01,{ }^{* *} p<0.001 ; N=182$.

Table 3 | Means and SD (in parentheses) of the EMO composite and two subscales across age (A) and sex (B).

\begin{tabular}{lllll}
\hline & $\begin{array}{l}\text { Number of } \\
\text { participants }\end{array}$ & $\begin{array}{l}\text { EMO } \\
\text { composite }\end{array}$ & $\begin{array}{l}\text { EMO } \\
\text { spatial }\end{array}$ & $\begin{array}{l}\text { EMO memory } \\
\text { for activities }\end{array}$ \\
\hline (A) AGE & & & & \\
8 & $N=17$ & $8.12(2.12)$ & $2.65(1.58)$ & $3.42(1.91)$ \\
9 & $N=18$ & $8.43(1.45)$ & $2.11(1.03)$ & $3.53(1.27)$ \\
10 & $N=16$ & $7.17(1.26)$ & $1.61(0.63)$ & $2.57(1.24)$ \\
11 & $N=21$ & $7.38(1.46)$ & $1.95(1.10)$ & $2.73(1.43)$ \\
12 & $N=19$ & $7.99(1.67)$ & $2.04(0.93)$ & $3.00(1.47)$ \\
13 & $N=18$ & $7.57(1.13)$ & $1.43(0.47)$ & $3.19(1.12)$ \\
14 & $N=14$ & $7.48(1.18)$ & $1.95(0.90)$ & $2.77(1.14)$ \\
15 & $N=18$ & $7.10(1.55)$ & $1.72(1.01)$ & $2.38(1.40)$ \\
16 & $N=15$ & $6.98(1.22)$ & $1.38(0.42)$ & $2.32(1.04)$ \\
(B) SEX & & & & \\
Males & $N=77$ & $7.89(1.57)$ & $2.14(1.20)$ & $3.17(1.44)$ \\
Females & $N=79$ & $7.30(1.43)$ & $1.62(0.71)$ & $2.63(1.31)$ \\
\hline
\end{tabular}

$E M Q$, everyday memory questionnaire; the EMO composite is the square root of all 28 items and higher scores on the EMQ indicate poorer everyday memory ability.

$B=-0.20, p=0.014$, and the subscales of spatial everyday memory, $\Delta F(1,154)=10.33, B=-0.25, p=0.002$, and memory for everyday activities, $\Delta F(1,154)=6.05, B=-0.20, p=0.015$. On each measure, females had fewer reported everyday memory difficulties than males (see Table 3B for means and SDs across sex).

In order to determine whether age-related changes in everyday memory performance corresponded with those found in episodic AM, the EMQ data were re-analyzed after first controlling for episodic AM (i.e., total episodic details in the Recall + Specific Probe condition). Results indicated that the effects of age and sex on everyday memory were significantly reduced to trendlevel effects (at $p<0.05$ ) for the EMQ composite and memory for everyday activities subscale, but not for the spatial everyday memory subscale $(p<0.01)$, after controlling for episodic AM.

\section{DISCUSSION}

This study examined the effects of age and sex on both episodic and semantic AM performance, as well as everyday memory ability, in a large sample of typically developing children and adolescents between 8 and 16 years of age. More specifically, the newly developed CAI was used to determine whether the number of episodic and semantic AM details recalled within a single autobiographical narrative increased across both childhood and adolescence. Our results were consistent with previous findings by Piolino et al. (2007) and Picard et al. (2009) showing gradual age-related increases in episodic AM across childhood. Importantly, our study also demonstrated for the first time that these improvements extend well into adolescence. In addition, our results revealed robust age-related improvements within each subcategory of episodic AM details (e.g., event, place, time, etc.), as well as for experimenter ratings of episodic qualities of $\mathrm{AM}$, indicating that the overall quality of children's episodic AM significantly improves across both childhood and adolescence.

Similar to Picard et al.'s (2009) results, we found significant age-related improvements in semantic AM (i.e., the semantic subcategory of non-episodic details) during late childhood and early adolescence. However, age accounted for a greater proportion of the variance in the number of total episodic details recalled than in the number of semantic details (and total non-episodic details) recalled across both Recall and Recall + Specific Probe conditions. Thus, our results indicated that both episodic and semantic AM improved significantly with age, but greater age-related changes were evident in episodic AM than semantic AM. Our findings therefore support a hierarchical model of AM that suggests that semantic AM develops earlier and provides a foundation for the later and more gradual development of episodic AM (Mishkin et al., 1997; Picard et al., 2009). To the extent that these findings are specific to episodic AM, they cannot be accounted for by differences in non-mnemonic factors such as verbal abilities that may themselves be sensitive to age and sex differences.

Several researchers have suggested that improvements in episodic AM across childhood and adolescence may be particularly associated with the prolonged maturation of the prefrontal cortex and its memory retrieval and executive processes (e.g., Levine, 2004; Piolino et al., 2007). In the present study, age effects were similar across the Recall and Recall + Specific Probe conditions, indicating that high retrieval support did not significantly reduce the age effect. Thus, age-related changes in episodic AM during childhood and adolescence may not be solely due to improvements in uncued retrieval and memory search operations associated with the prefrontal cortex but may also depend on maturation of the hippocampus and the entire AM neural network, as well as other 
cognitive functions. For example, Picard et al. (2009) showed that episodic AM development is associated not only with improved executive functioning, which relies on the prefrontal cortex, but also with improved general episodic memory, which relies on the medial temporal lobes and other structures. In addition, increases in both gray matter volume and synaptic connectivity within the hippocampus have been observed across childhood and adolescence (Benes et al., 1994; Huttenlocher and Dabholkar, 1997; Grieve et al., 2011) and could contribute to age-related improvements in episodic AM. Given that AM relies on several diverse cognitive processes, as well as an entire network of brain regions (Svoboda et al., 2006), it is likely that numerous factors account for age-related changes in episodic AM across childhood and adolescence. Thus, additional investigations using both behavioral and neuroimaging techniques are required in order to investigate structure-function relations associated with episodic and semantic AM during childhood and adolescence.

The present study also represents the first examination of sex differences in both episodic and semantic AM across childhood and adolescence. The results confirmed previous findings in other age groups, as females were found to recall more total episodic, event, time, and perceptual details than males in the Recall condition. Importantly, these sex differences in episodic AM recall were reduced after the provision of high retrieval support (i.e., the Specific Probe phase). This finding corresponds to Bloise and Johnson's (2007) claim that males may have less efficient prefrontal memory retrieval and organizational processes than females, especially during effortful and unstructured retrieval tasks with low retrieval support (e.g., the Recall condition); however, this issue warrants further investigation. Similar to previous studies, we also found that females recalled a greater number of emotion/thought details than did males in the Recall + Specific Probe condition. This finding of a sex difference in emotion/thought details in the Recall + Specific Probe condition, but not in the Recall condition, likely reflects the fact that participants were specifically asked to recall emotions/thoughts during the Specific Probe phase, but not during the Recall phase. Most importantly, no significant sex differences were found in semantic AM (i.e., total semantic details recalled), indicating for the first time that sex differences in AM appear to be evident only in the episodic component of AM.

Based on participants' self-report ratings, we found that participants' ability to visualize each event during recollection is strongly associated with the overall quality of their episodic AM recollections. For instance, greater visualization during recollection was significantly associated with a greater number of episodic details recalled, particularly within the perceptual subcategory of details. This finding supports the idea that visual imagery is an important feature of episodic AM retrieval (Rubin and Greenberg, 1998; Addis et al., 2004). Interestingly, previous neuroimaging studies have shown that vividly re-experienced AMs are associated with increased hippocampal activation in adults (e.g., Gilboa et al., 2004), suggesting a specific role for the hippocampus in both re-experiencing and visualizing past events.

Given that normal hippocampal functioning is essential for both episodic AM and everyday memory (Vargha-Khadem et al., 1997; Isaacs et al., 2000), we also examined the effects of age and sex on everyday memory in order to determine whether the results would be similar to those observed in episodic AM. Unlike Drysdale et al.'s (2004) results, we observed that age and sex both significantly predicted everyday memory ability, specifically memory for everyday activities and spatial everyday memory. In addition, the effects of age and sex on everyday memory, particularly memory for everyday activities, were significantly reduced after first controlling for episodic AM (i.e., the number of total episodic details recalled). This finding suggests that age-related increases in memory for everyday activities correspond with agerelated increases in episodic AM, possibly because these abilities are dependent on the maturation of similar brain regions (e.g., the hippocampus) or neural networks. However, further investigation of the relation between episodic AM and everyday memory using longitudinal data and neuroimaging techniques is required.

Several important limitations of the present study warrant discussion. First, we were not able to verify the details of participants' AMs in order to determine whether they accurately represented the original event details. It is often difficult to assess the accuracy of children's AMs without creating a staged autobiographical event, given that parental verification of event details can also be inaccurate or biased and parents are unable to verify details from events in which they were not present (e.g., sleepovers or school trips). However, because our sample size is relatively large and our results replicate previous findings, we believe that our results accurately represent age-related changes and sex differences in the ability to recall episodic and semantic AM details. Second, due to the cross-sectional design of the present study, we were not able to identify individual developmental trends in episodic and semantic AM. Therefore, our conclusions concerning agerelated changes in episodic and semantic AM, as well as everyday memory, should be interpreted with caution. Clearly, longitudinal studies of episodic and semantic AM are required to further investigate these developmental trajectories. Finally, because the current study was designed to explore AM in a time-limited fashion with a convenience sample of children, we could not administer control measures or additional tests laboratory tests that might have provided valuable additional information about the mechanisms underlying the observed effects in AM.

In summary, the present study used the newly developed CAI to provide a detailed account of age-related changes and sex differences in episodic and semantic AM across childhood and adolescence. Although a considerable amount of research on AM has been conducted to date, most studies so far have not investigated episodic and semantic AM together, especially during late childhood and adolescence. Thus, the present study provides important new insight into episodic and semantic AM, as well as the relation between episodic AM and everyday memory during this often neglected developmental time period. In addition, our results indicate for the first time that sex differences in AM are only evident in the episodic component of AM. The critical contribution of this study, however, is to provide normative data using the CAI on developmental changes in episodic and semantic memory components from childhood through to adolescence. In order to improve our understanding of the underlying neural mechanisms that support episodic and semantic AM retrieval and how they develop across childhood and adolescence, further research using neuroimaging techniques is required. In addition, investigations 
into episodic and semantic AM in atypically developing children, such as children with early hippocampal damage, using the CAI would be particularly useful for determining how abnormal brain development affects episodic and semantic AM performance.

\section{ACKNOWLEDGMENTS}

We would like to thank Dr. Mary Pat McAndrews, Dr. Erin Sheard, Dr. Sarah Wheeler, and Jovanka Skocic for their invaluable help

\section{REFERENCES}

Addis, D. R., Moscovitch, M., Crawley, A. P., and McAndrews, M. P. (2004). Recollective qualities modulate hippocampal activation during autobiographical memory retrieval. Hippocampus 14, 752-762.

Addis, D. R., Moscovitch, M., and McAndrews, M. P. (2007). Consequences of hippocampal damage across the autobiographical memory network in left temporal lobe epilepsy. Brain 130, 2327-2342.

Bachevalier, J., and Vargha-Khadem, F. (2005). The primate hippocampus: ontogeny, early insult and memory. Curr. Opin. Neurobiol. 15, 168-174.

Bahrick, H. P., Bahrick, P. O., and Wittlinger, R. P. (1975). Fifty years of memory for names and faces: a cross-sectional approach. J. Exp. Psychol. Gen. 104, 54-75.

Barr, R., Dowden, A., and Hayne, H. (1996). Developmental changes in deferred imitation by 6- to 24month-old infants. Infant Behav. Dev. 19, 159-170.

Bauer, P. J. (2006). Constructing a past in infancy: a neuro-developmental account. Trends Cogn. Sci. (Regul. Ed.) 10, 175-181.

Bauer, P. J., Burch, M. M., Scholin, S. E., and Güler, O. E. (2007). Using cue-words to investigate the distribution of autobiographical memories in childhood. Psychol. Sci. 18, 910-916.

Bauer, P. J., and Dow, G. A. (1994). Episodic memory in 16- and 20month-old children: specifics are generalized, but not forgotten. Dev. Psychol. 30, 403-417.

Benes, F. M., Turtle, M., Khan, Y., and Farol, P. (1994). Myelination of a key relay zone in the hippocampal formation occurs in the human brain during childhood, adolescence, and adulthood. Arch. Gen. Psychiatry 51, 477-484.

Bloise, S. M., and Johnson, M. K. (2007). Memory for emotional and neutral information: gender and individual differences in emotional sensitivity. Memory 15, I192-I204.

Bruce, D., Dolan, A., and Phillips-Grant, K. (2000). On the transition from childhood amnesia to the recall of personal memories. Psychol. Sci. 11, 360-364.

Buckner, J. P., and Fivush, R. (1998). Gender and self in children's autobiographical narratives. Appl. Cogn. Psychol. 12, 407-429.

Buckner, R. L., and Wheeler, M. E. (2001). The cognitive neuroscience of remembering. Nat. Rev. Neurosci. 2, 624-634.

Cabeza, R., Prince, S. E., Daselaar, S. M., Greenberg, D. L., Budde, M., Dolcos, F., LaBar, K. S., and Rubin, D. C. (2004). Brain activity during episodic retrieval of autobiographical and laboratory events: an fMRI study using a novel photo paradigm. J. Cogn. Neurosci. 16, 1583-1594.

Conway, M. A., and Pleydell-Pearce, C. W. (2000). The construction of autobiographical memories in the selfmemory system. Psychol. Rev. 107, 261-288.

Cornish, I. M. (2000). Factor structure of the everyday memory questionnaire. Br. J. Psychol. 91, 427-438.

Davis, P. J. (1999). Gender differences in autobiographical memory for childSoc. Psychol. 76, 498-510.

Drysdale, K., Shores, A., and Levick, W. (2004). Use of the everyday memory questionnaire with children. Child Neuropsychol. 10, 67-75.

Eichenbaum, H. (2000). A corticohippocampal system for declarative memory. Nat. Rev. Neurosci. 1, 41-50.

Eichenbaum, H., and Bunsey, M. (1995). On the binding of associations in memory: clues from studies on the role of the hippocampal region in paired-associate learning. Curr. Dir. Psychol. Sci. 4, 19-23.

Eldridge, L. L., Knowlton, B. J., Furmanski, C. S., Bookheimer, S. Y., and Engel, S. A. (2000). Remembering episodes: a selective role for the hippocampus during retrieval. Nat. Neurosci. 3, 1149-1152.

Fivush, R., and Hamond, N. R. (1990). "Autobiographical memory across the preschool years: toward reconceptualizing childhood amnesia," in Knowing and Remembering in Young hood emotional experiences. J. Pers.

and support for the present study. We would also like the volunteers who helped with transcribing and scoring. We are most grateful to the Ontario Science Centre, as well as to the many parents/caregivers and their children for their participation in this study. This work was supported by a Canadian Institutes of Health Research (CIHR) grant, as well as an Ontario Graduate Scholarship, and a Hospital for Sick Children RESTRACOMP scholarship.

Children, eds R. Fivush and J. A. Hudson (Cambridge, NY: University Press), 223-248.

Giedd, J. N., Vaituzis, A. C., Hamburger, S. D., Lange, N., Rajapakse, J. C., Kaysen, D., Vauss, Y. C., and Rapoport, J. L. (1996). Quantitative MRI of the temporal lobe, amygdala, and hippocampus in normal human development: ages 4-18 years. J. Comp. Neurol. 366, 223-230.

Gilboa, A., Winocur, G., Grady, C. Hevenor, S. J., and Moscovitch, M. (2004). Remembering our past: functional neuroanatomy of recollection of recent and very remote personal events. Cereb. Cortex 14, 1214-1225.

Gogtay, N., Nugent, T. F., Herman, D. H., Ordonez, A., Greenstein, D., Hayashi, K. M., Clasen, L., Toga, A. W., Giedd, J. N., Rapoport, J. L., and Thompson, P. M. (2006). Dynamic mapping of normal human hippocampal development. Hippocampus 16, 664-672.

Grieve, S. M., Korgaonkar, M. S., Clark, C. R., and Williams, L. M. (2011). Regional heterogeneity in limbic maturational changes: evidence from integrating cortical thickness, volumetric, and diffusion tensor imaging measures. Neuroimage 55, 868-879.

Herlitz, A., Nilsson, L. G., and Backman, L. (1997). Gender differences in episodic memory. Mem. Cognit. 25, 801-811.

Hollingshead, A. B. (1975). Four-Factor Index of Social Status. New Haven, CT: Yale University.

Hoscheidt, S. M., Nadel, L., Payne, J., and Ryan, L. (2010). Hippocampal activation during retrieval of spatial context from episodic and semantic memory. Behav. Brain Res. 212, 121-132.

Howe, M. L., and Courage, M. L. (1993). On resolving the enigma of infantile amnesia. Psychol. Bull. 113, 305-326.

Howe, M. L., and Courage, M. L. (1997). The emergence and early development of autobiographical memory. Psychol. Rev. 104, 499-523.

Howe, M. L., Courage, M. L., and Edison, S. C. (2003). When autobio- graphical memory begins. Dev. Rev. 23, 471-494.

Hudson, J. A., and Fivush, R. (1991). As time goes by: sixth graders remember a kindergarten experience. Appl. Cogn. Psychol. 5, 347-360.

Huttenlocher, P. R., and Dabholkar, A. S. (1997). Regional differences in synaptogenesis in human cerebral cortex. J. Comp. Neurol. 387, 167-178.

Isaacs, E. B., Lucas, A., Chong, W. K., Wood, S. J., Johnson, C. L., Marshall, C., Vargha-Khadem, F., and Gadian, D. G. (2000). Hippocampal volume and everyday memory in children of very low birth weight. Pediatr. Res. 47, 713-720.

Kopelman, M. D., Wilson, B. A., and Baddeley, A. D. (1989). The autobiographical memory interview: a new assessment of autobiographical and personal semantic memory in amnesic patients. J. Clin. Exp. Neuropsychol. 11, 724-744.

Lechuga, M. T., Marcos-Ruiz, R., and Bauer, P. J. (2001). Episodic recall of specifics and generalization coexist in 25-month-old children. Memory 9, 117-132.

Lenroot, R. K., and Giedd, J. N. (2006). Brain development in children and adolescents: insights from anatomical magnetic resonance imaging. Neurosci. Biobehav. Rev. 30, 718-729.

Levine, B. (2004). Autobiographical memory and the self in time: brain lesion effects, functional neuroanatomy, and lifespan development. Brain Cogn. 55, 54-68.

Levine, B., Svoboda, E., Hay, J. F., Winocur, G., and Moscovitch, M. (2002). Aging and autobiographical memory: dissociating episodic from semantic retrieval. Psychol. Aging 17, 677-689.

Levine, B., Turner, G., Tisserand, D., Hevenor, S. J., Graham, S. J., and McIntosh, A. R. (2004). The functional neuroanatomy of episodic and semantic autobiographical remembering: a prospective functional MRI study. J. Cogn. Neurosci. 16, 1633-1646.

Maguire, E. A., and Mummery, C. J. (1999). Differential modulation of 
a common memory retrieval network revealed by positron emission tomography. Hippocampus 9, 54-61.

Maguire, E. A., Vargha-Khadem, F., and Mishkin, M. (2001). The effects of bilateral hippocampal damage on fMRI regional activations and interactions during memory retrieval. Brain 124, 1156-1170.

McGraw, K. O., and Wong, S. P. (1996). Forming inferences about some intraclass correlation coefficients. Psychol. Methods 1, 30-46.

Meltzoff, A. N. (1995). What infant memory tells us about infantile amnesia: long-term recall and deferred imitation. J. Exp. Child. Psychol. 59, 497-515.

Mishkin, M., Suzuki, W. A., Gadian, D. G., and Vargha-Khadem, F. (1997). Hierarchical organization of cognitive memory. Philos. Trans. R. Soc. Lond. B Biol. Sci. 352, 1461-1467.

Moscovitch, M. (2008). The hippocampus as a "stupid," domain-specific module: implications for theories of recent and remote memory, and of imagination. Can. J. Exp. Psychol. 62, 62-79.

Nelson, K., and Fivush, R. (2004). The emergence of autobiographical memory: a social cultural developmental theory. Psychol. Rev. 111, 486-511.

Newcombe, N. S., Lloyd, M. E., and Ratliff, K. R. (2007). "Development of episodic and autobiographical memory: a cognitive neuroscience perspective," in Advances in Child Development and Behavior, Vol. 35, ed. R. V. Kail (San Diego, CA: Elsevier), 37-85.

Perner, J., and Ruffman, T. (1995). Episodic memory and autonoetic consciousness: developmental evidence and the theory of childhood amnesia. J. Exp. Child Psychol. 59, 516-548.
Picard, L., Reffuveille, I., Eustache, F., and Piolino, P. (2009). Development of autonoetic autobiographical memory in school-age children: genuine age effect or development of basic cognitive abilities? Conscious Cogn. 18, 864-876.

Pillemer, D. B., Picariello, M. L., and Pruett, J. C. (1994). Very long-term memories of a salient preschool event. Appl. Cogn. Psychol. 8, 95-106.

Pillemer, D. B., Wink, P., DiDonato, T., and Sanborn, R. L. (2003). Gender differences in autobiographical memory styles of older adults. Memory $11,525-532$.

Piolino, P., Hisland, M., Ruffeveille, I., Matuszewski, V., Jambaqué, I., and Eustache, F. (2007). Do school-age children remember or know the personal past? Conscious Cogn. 16, 84-101.

Ross, M., and Holmberg, D. (1992). Are wives' memories for events in relationships more vivid than their husbands' memories? J. Soc. Pers. Relat. 9, 585-604.

Rubin, D. C. (2000). The distribution of early childhood memories. Memory 8, 265-269.

Rubin, D. C., and Greenberg, D. L. (1998). Visual memory-deficit amnesia: a distinct amnesic presentation and etiology. Proc. Natl. Acad. Sci. U.S.A. 95, 5413-5416.

Ryan, L., Nadel, L., Keil, K., Putman, K., Schnyer, D., Trouard, T., and Moscovitch, M. (2001). Hippocampal complex and retrieval of recent and very remote autobiographical memories: evidence from functional magnetic resonance imaging in neurologically intact people. Hippocampus 11, 707-714.

Steinvorth, S., Levine, B., and Corkin, S. (2005). Medial temporal lobe structures are needed to re-experience remote autobiographical memories: evidence from H.M. and W.R. Neuropsychologia 43, 479-496.

St-Laurent, M., Moscovitch, M., Levine, B., and McAndrews, M. P. (2009) Determinants of autobiographical memory in patients with unilateral temporal lobe epilepsy or excisions. Neuropsychologia 47, 2211-2221.

Sunderland, A., Harris, J. E., and Baddeley, A. D. (1983). "Assessing everyday memory after severe head injury," in Everyday Memory, Actions, and Absent-Mindedness, eds J. E. Harris and P. E. Morris (London: Academic Press), 191-206.

Suzuki, M., Hagino, H., Nohara, S. Zhou, S., Kawasaki, Y., Takahashi, T., Matsui, M., Seto, H., Ono, T., and Kurachi, M. (2005). Male-specific volume expansion of the human hippocampus during adolescence. Cereb. Cortex 15, 187-193.

Svoboda, E., Hynes, C. A., Campbell, A. F., Dade, L. A., Moscovitch, M., and Levine, B. (2002). The frontal lobes and autobiographical memory: differential effects of dorsolateral and ventrolateral prefrontal damage. $J$. Int. Neuropsychol. Soc. 8, 275.

Svoboda, E., McKinnon, M. C., and Levine, B. (2006). The functional neuroanatomy of autobiographical memory: a meta-analysis. Neuropsychologia 44, 2189-2208.

Tulving, E. (2002). Episodic memory: from mind to brain. Annu. Rev. Psychol. 53, 1-25.

Van Abbema, D. L., and Bauer, P. J. (2005). Autobiographical memory in middle childhood: recollections of the recent and distant past. Memory $13,829-845$.

Vargha-Khadem, F., Gadian, D. G. Watkins, K. E., Connelly, A., Van Paesschen, W., and Mishkin, M. (1997). Differential effects of early hippocampal pathology on episodic and semanticmemory. Science 277 , 376-380.
Viskontas, I. V., McAndrews, M. P., and Moscovitch, M. (2000). Remote episodic memory deficits in patients with unilateral temporal lobe epilepsy and excisions. $J$. Neurosci. 20, 5853-5857.

Wheeler, M. A., Stuss, D. T., and Tulving, E. (1997). Toward a theory of episodic memory: the frontal lobes and autonoetic consciousness. Psychol. Bull. 121, 331-354.

Willoughby, K. A., Sheard, E. D., Nash, K., and Rovet, J. (2008). Effect of prenatal alcohol exposure on Hippocampal volume, verbal learning, and verbal and spatial recall in late childhood. J. Int. Neuropsychol. Soc. 14, 1022-1033.

Conflict of Interest Statement: The authors declare that the research was conducted in the absence of any commercial or financial relationships that could be construed as a potential conflict of interest.

Received: 12 July 2011; accepted: 10 February 2012; published online: 28 February 2012.

Citation: Willoughby KA, Desrocher $M$, Levine B and Rovet JF (2012) Episodic and semantic autobiographical memory and everyday memory during late childhood and early adolescence. Front. Psychology 3:53. doi: 10.3389/fpsyg.2012.00053

This article was submitted to Frontiers in Developmental Psychology, a specialty of Frontiers in Psychology.

Copyright (c) 2012 Willoughby, Desrocher, Levine and Rovet. This is an open-access article distributed under the terms of the Creative Commons Attribution Non Commercial License, which permits non-commercial use, distribution, and reproduction in other forums, provided the original authors and source are credited. 


\section{APPENDIX \\ INSTRUCTIONS FOR THE CHILDREN'S AUTOBIOGRAPHICAL INTERVIEW}

"I am going to ask you to tell me about two events that have happened to you in the past. I will give you a list of some events that might be of help. You can choose two events from this list or you can choose a different event, one that is not on the list. There are three rules to follow when you are choosing your two events. First, you can pick an event from any time in your life, as long as it happened more than 1 month ago because I do not want you to describe a very recent event. The second rule is that the events that you choose have to be ones where you were personally there and you took part in what happened. Do not pick events that you have only heard about from your parents, family, or friends, or only saw in a photograph, they must have happened to you. Finally, the third rule is that I want you to pick an event that happened at a specific time and place. You should pick an event that happened within a couple hours or 1 day at the most. For example, I do not want you to describe a 3-week long vacation because that is not specific enough, however, you could tell me about something that happened on 1 day during your vacation.

I will ask you to describe one event first, then the other one. Then I will ask you some questions about the events. I would like you to give me as much detail on what happened as you can. I am not interested in which events you choose, but I am interested in how you tell the event to me. So, try and pick events that you feel comfortable describing to me in detail. To help me remember what you said, I will be audiotaping your description of the event and your answers to the questions, but because your name is not on the tape it will be completely confidential and it will be stored in a locked cabinet so that no one else has access to it. Do you have any questions?" 
Table A1 | Regressions with age and sex predicting episodic and non-episodic details in the Recall condition, after controlling for retention interval.

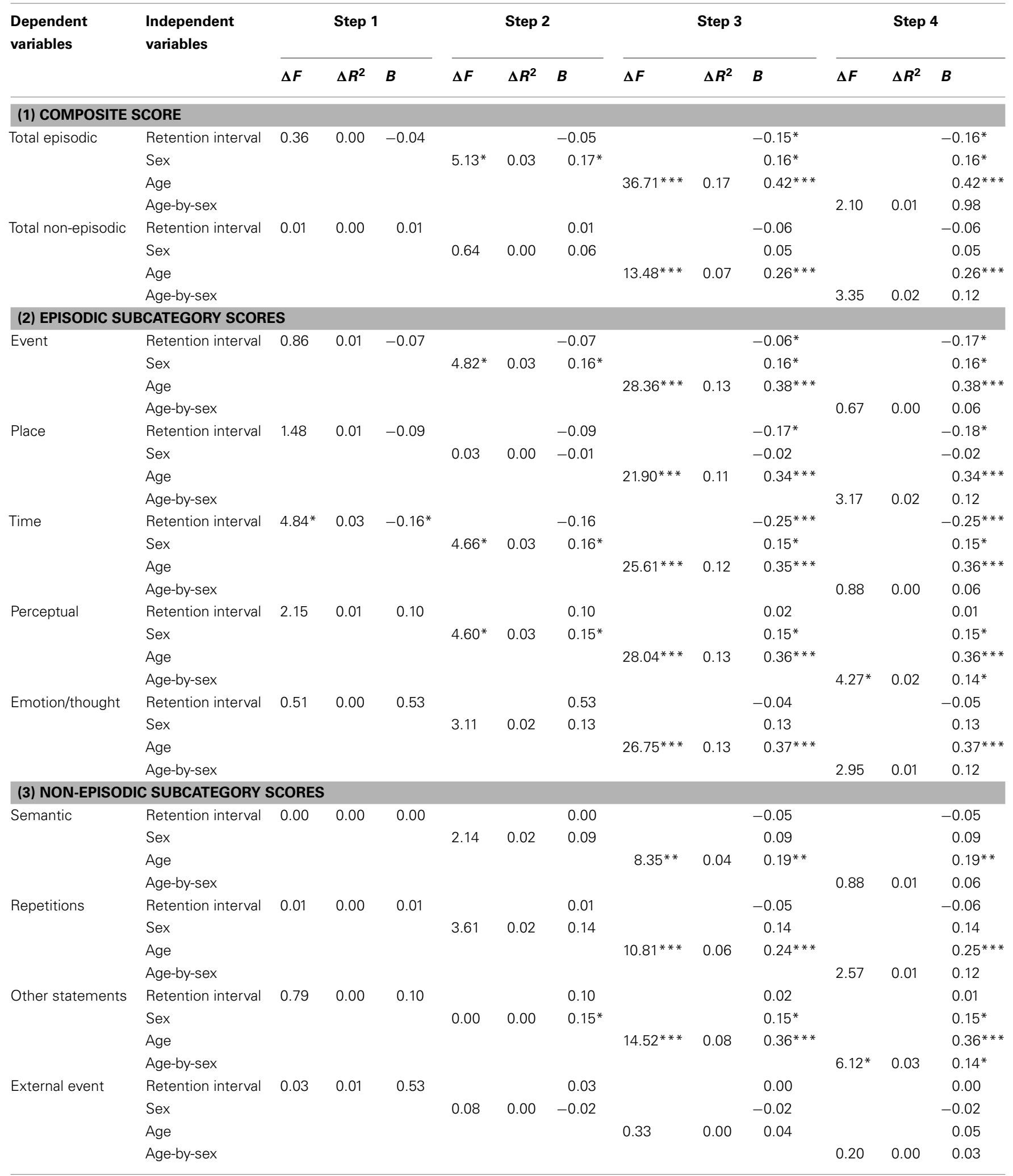

$B$, unstandardized regression coefficient; ${ }^{*} p<0.05,{ }^{* *} p<0.01,{ }^{* * *} p<0.001$. 
Table A2 | Regressions with age and sex predicting episodic and non-episodic details in the recall + specific probe condition, after controlling for retention interval.

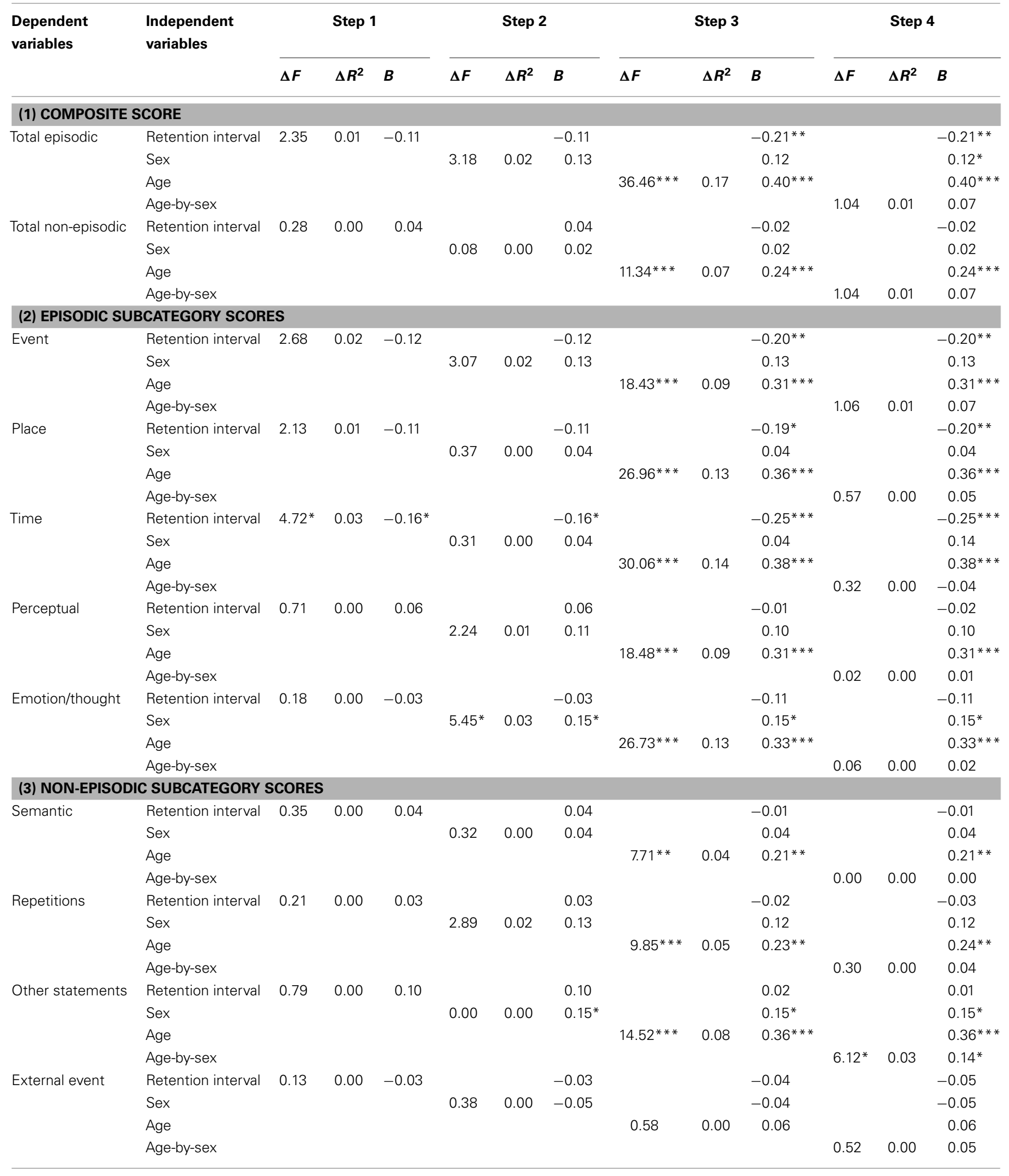


Table A2 | Continued

\begin{tabular}{|c|c|c|c|c|c|c|c|c|c|c|c|c|c|}
\hline \multirow{2}{*}{$\begin{array}{l}\text { Dependent } \\
\text { variables }\end{array}$} & \multirow{2}{*}{$\begin{array}{l}\text { Independent } \\
\text { variables }\end{array}$} & \multicolumn{3}{|c|}{ Step 1} & \multicolumn{3}{|c|}{ Step 2} & \multicolumn{3}{|c|}{ Step 3} & \multicolumn{3}{|c|}{ Step 4} \\
\hline & & $\Delta F$ & $\Delta R^{2}$ & $B$ & $\Delta \boldsymbol{F}$ & $\Delta R^{2}$ & $B$ & $\Delta F$ & $\Delta R^{2}$ & $B$ & $\Delta \boldsymbol{F}$ & $\Delta R^{2}$ & $B$ \\
\hline \multicolumn{14}{|c|}{ (4) EXPERIMENTER RATINGS } \\
\hline \multirow[t]{2}{*}{ Rating composite } & Retention interval & 0.78 & 0.00 & -0.07 & & & -0.07 & & & $-0.15^{*}$ & & & -0.15 \\
\hline & Sex & & & & 2.53 & 0.01 & 0.12 & & & 0.11 & & & 0.11 \\
\hline \multirow[t]{4}{*}{ Time integration } & Retention interval & 0.00 & 0.00 & -0.01 & & & -0.01 & & & -0.11 & & & -0.11 \\
\hline & Sex & & & & 2.27 & 0.01 & 0.11 & & & 0.11 & & & 0.11 \\
\hline & Age & & & & & & & $35.09 * * *$ & 0.16 & $0.42 * * *$ & & & $0.42 * * *$ \\
\hline & Age-by-sex & & & & & & & & & & 1.14 & 0.01 & 0.07 \\
\hline
\end{tabular}

$B$, unstandardized regression coefficient; ${ }^{*} p<0.05,{ }^{*} p<0.01,{ }^{*}{ }^{*} p<0.001$. 